\title{
MHD FLOW PAST A PARABOLIC STARTED VERTICAL PLATE WITH VARIABLE TEMPERATURE AND MASS DIFFUSION
}

\author{
A. Neel Armstrong ${ }^{1,2}$ and R. Muthucumaraswamy, \\ ${ }^{1}$ Faculty of Mathematics, SKR Engineering College / Anna University, \\ Poonamalle / Chennai, Tamil Nadu, India - 600123 \\ ${ }^{2}$ Research and Development Centre, \\ Department of Mathematics, Manonmaniam Sundaranar University, \\ Tirunelveli, Tamil Nadu, India - 627012 \\ ${ }^{3}$ Department of Applied Mathematics, \\ Sri Venkateswara College of Engineering / Anna University, \\ Pennalur, Irungattukottai, Sriperumbudur Taluk / Chennai, \\ Tamil Nadu, India - 602117 \\ * Email: msamy@svce.ac.in \\ Phone: +9144-27152000; Fax: +9144-27162462
}

\begin{abstract}
The theoretical analysis of magnetohydrodynamic flow past a parabolic motion started infinite vertical plate with variable temperature and uniform mass diffusion has been analysed. The plate temperature is raised linearly with time and the concentration level near the plate is raised uniformly. The dimensionless governing equations are solved using the Laplace transform technique. The effect of the velocity profile is discussed for different physical parameters like the thermal Grashof number, mass Grashof number, Schmidt number, and time. We conclude that the observation of velocity increases as the value of the thermal Grashof number or mass Grashof number increases. The trend is reversed with respect to the magnetic field parameter, as shown with the help of graphs.
\end{abstract}

Keywords: variable temperature; parabolic; vertical plate; uniform mass diffusion; Laplace transform.

\section{INTRODUCTION}

Magnetohydrodynamics (MHD) is the discipline which studies the dynamics of electrically conducting fluids. The main idea of MHD is that magnetic fields can induce currents in a moving conductive fluid, which creates forces on the fluid, and also changes the magnetic field parameter 
[1-4]. The set of equations which describe MHD is a combination of the Navier-Stokes equations of fluid dynamics and Maxwell's equations of electromagnetism. MHD describes the two main applications of plasma physics, thermo-nuclear fusion energy and plasma astrophysics of the solar system, including stars, the interplanetary medium (space between the planets), the interstellar medium (space between the stars), the intergalactic medium, nebulae and jets [5]. Many astrophysical systems are not in thermal equilibrium, and this therefore requires an additional kinematic treatment to describe all the phenomena within the system. The solar wind is also governed by MHD [6]. This approach provides effective methods and insights for the interpretation of plasma phenomena on virtually all scales, from the laboratory to the universe. An MHD drive or MHD propulsor is a method for propelling seagoing vessels using only electric and magnetic fields with no moving parts. The working principle involves electrification of the propellant (gas or water), which can then be directed by a magnetic field, pushing the vehicle in the opposite direction. MHD is used to stabilize a flow against the transition from laminar to turbulent flow [7]. The effect of the magnetic field on the viscous incompressible flow of electrically conducting fluid is important in many applications, such as extrusion of plastics in the manufacture of rayon and nylon, purification of crude oil, pulp, the paper industry, textile industry and in different geophysical cases, etc. Magneto-convection plays an important role in agriculture, the petroleum industries, geophysics and in astrophysics. There are important applications in the study of geological formations, in exploration and thermal recovery of oil, and in the assessment of aquifers, geothermal reservoirs and underground nuclear waste storage sites. MHD flow has applications in metrology, solar physics and in the motion of the earth's core. Also, it has applications in the field of stellar and planetary magnetospheres, aeronautics, chemical engineering and electronics. In many process industries, the cooling of threads or sheets of some polymer materials is of importance in the production line. The rate of cooling or heating can be controlled effectively to achieve the desired characteristics by drawing threads, etc.

Gupta et al. [8] studied free convection effects on the flow past an accelerated vertical plate in an incompressible dissipative fluid. Raptis and Singh [9] investigated MHD free convection flow past an accelerated vertical plate. Soundalgekar [10] reported on the effects of mass transfer on flow past a uniformly accelerated vertical plate. The mass transfer effects on flow past an accelerated vertical plate with heat flux were analysed also by Singh and Singh [5]. Jha and Prasad [11] analysed free convection and mass transfer effects on flow past an accelerated vertical plate with heat sources. Agrawal et al. [12] studied heat and mass transfer flow past a 
parabolic started infinite vertical plate. Hence, it is proposed to study hydromagnetic effects on flow past a parabolic infinite vertical plate with variable temperature and uniform mass diffusion subject to parabolic motion in the presence of magnetic field. The dimensionless governing equations are solved using the Laplace transform technique. The solutions are in terms of exponential and complementary error function. Such a study is found useful in magnetic control of molten iron flow in the steel industry, liquid metal cooling in nuclear reactors, magnetic suppression of molten semi-conducting materials and meteorology.

\section{MATHEMATICAL FORMULATION}

In this paper the unsteady flow of a viscous incompressible fluid past an infinite vertical plate with variable temperature and uniform mass diffusion in the presence of magnetic field has been considered. Here the unsteady flow of a viscous incompressible fluid is initially at rest and surrounds an infinite vertical plate with temperature $T_{\infty}$ and concentration $C_{\infty}^{\prime}$. The xaxis is taken along the plate in the vertically upward direction and the $y$ axis is taken normal to the plate. At time $t^{\prime} \leq 0$, the plate and fluid are at the same temperature $T_{\infty}$ and concentration $C_{\infty}^{\prime}$. At time $t^{\prime}>0$, the plate is parabolic started with a velocity $u=u_{0} t^{\prime 2}$ in its own plane against gravitational field, and the temperature from the plate is raised to $T_{w}$ and the concentration level near the plate is also raised to $C_{w}^{\prime}$ with time $t$ and the mass is diffused uniformly from the plate to the fluid. Since the plate is infinite in length, all the terms in the governing equations will be independent of $x$ and there is no flow along the y-direction. The infinite vertical plate is also subjected to a uniform magnetic field of strength $B_{0}$ which is assumed to be applied normal to the plate. Then the unsteady flow is governed by free-convective flow of an electrically conducting fluid in a parabolic started motion under the usual Boussinesq's and boundary layer approximation, which is governed by the following dimensionless form of equations:

$$
\begin{gathered}
\frac{\partial u}{\partial t^{\prime}}=g \beta\left(T-T_{\infty}\right)+g \beta^{*}\left(C^{\prime}-C_{\infty}^{\prime}\right)+v \frac{\partial^{2} u}{\partial y^{2}}-\frac{\sigma B_{0}^{2}}{\rho} u \\
\rho C_{P} \frac{\partial T}{\partial t^{\prime}}=k \frac{\partial^{2} T}{\partial y^{2}}
\end{gathered}
$$




$$
\frac{\partial C^{\prime}}{\partial t^{\prime}}=D \frac{\partial^{2} C^{\prime}}{\partial y^{2}}
$$

with the following initial and boundary conditions:

$$
\begin{aligned}
& u=0 \quad, \quad T=T_{\infty} \quad C^{\prime}=C_{\infty}^{\prime} \quad \text { for all } y, t^{\prime} \leq 0 \\
& t^{\prime}>0: \quad u=u_{0} t^{\prime 2}, \quad T=T_{\infty}+\left(T_{w}-T_{\infty}\right) A t^{\prime}, \quad C^{\prime}=C_{w}^{\prime} \quad \text { at } \quad y=0 \\
& u \rightarrow 0, \quad T \rightarrow T_{\infty}, \quad C^{\prime} \rightarrow C_{\infty}^{\prime} \quad \text { as } \quad y \rightarrow \infty
\end{aligned}
$$

where $A=\frac{u_{0}^{2}}{v}$

On introducing the following non-dimensional quantities:

$$
\begin{gathered}
U=u\left(\frac{u_{0}}{v^{2}}\right)^{1 / 3}, \quad t=\left(\frac{u_{0}^{2}}{v}\right)^{1 / 3} t^{\prime}, \quad Y=y\left(\frac{u_{0}}{v^{2}}\right)^{1 / 3} \\
\theta=\frac{T-T_{\infty}}{T_{w}-T_{\infty}}, \quad G r=\frac{g \beta\left(T_{w}-T_{\infty}\right)}{\left(v u_{0}\right)^{1 / 3}} \\
C=\frac{C^{\prime}-C_{\infty}^{\prime}}{C_{w}^{\prime}-C_{\infty}^{\prime}}, \quad G c=\frac{g \beta\left(C^{\prime}{ }_{w}-C_{\infty}^{\prime}\right)}{\left(v u_{0}\right)^{1 / 3}} \\
M=\frac{\sigma B_{0}^{2}}{\rho}\left(\frac{v}{u_{0}^{2}}\right)^{\frac{1}{3}}, \quad \operatorname{Pr}=\frac{\mu C_{p}}{k}, \quad S c=\frac{v}{D}
\end{gathered}
$$

in Eqs. (1)-(4), leads to

$$
\begin{aligned}
\frac{\partial U}{\partial t} & =G r \theta+G c C+\frac{\partial^{2} U}{\partial Y^{2}}-M U \\
\frac{\partial \theta}{\partial t} & =\frac{1}{\operatorname{Pr}} \frac{\partial^{2} \theta}{\partial Y^{2}} \\
\frac{\partial C}{\partial t} & =\frac{1}{S c} \frac{\partial^{2} C}{\partial Y^{2}}
\end{aligned}
$$


The initial and boundary conditions in non-dimensional quantities are

$$
\begin{aligned}
& U=0, \theta=0, \quad C=0 \quad \text { for all } Y, t \leq 0 \\
& t>0: U=t^{2}, \quad \theta=\mathrm{t}, \quad C=1 \quad \text { at } \quad Y=0 \\
& U \rightarrow 0, \quad \theta \rightarrow 0, \quad C \rightarrow 0 \quad \text { as } \quad Y \rightarrow \infty
\end{aligned}
$$

The non-dimensional quantities are defined in the nomenclature.

\section{METHOD OF SOLUTION}

The dimensionless governing Eqs. (6)-(8), subject to the corresponding initial and boundary conditions [13], are tackled using the Laplace transform technique and the solutions are derived as follows:

$$
\begin{gathered}
\theta=\mathrm{t}\left(\left(1+2 \eta^{2} \operatorname{Pr}\right) \operatorname{erfc}(\eta \sqrt{\operatorname{Pr}})-2 \frac{\eta \sqrt{\operatorname{Pr}}}{\sqrt{\pi}} \exp \left(-\eta^{2} \operatorname{Pr}\right)\right) \\
\mathrm{C}=\operatorname{erfc}(\eta \sqrt{\mathrm{Sc}}) \\
U=2 *\left[\left(\frac{\left(\eta^{2}+\mathrm{Mt}\right) \mathrm{t}}{4 \mathrm{M}}[\exp (2 \eta \sqrt{\mathrm{Mt}}) \operatorname{erfc}(\eta+\sqrt{\mathrm{Mt}})+\exp (-2 \eta \sqrt{\mathrm{Mt}}) \operatorname{erfc}(\eta-\sqrt{\mathrm{Mt}})]\right]\right. \\
+\frac{\eta \sqrt{\mathrm{t}}(1-4 \mathrm{Mt})}{8 \mathrm{M}^{3 / 2}}[\exp (-2 \eta \sqrt{\mathrm{Mt}}) \operatorname{erfc}(\eta-\sqrt{\mathrm{Mt}})-\exp (2 \eta \sqrt{\mathrm{Mt}}) \operatorname{erfc}(\eta+\sqrt{\mathrm{Mt}})] \\
\left.-\frac{\eta t}{2 \mathrm{M} \sqrt{\pi}} \exp \left(-\left(\eta^{2}+\mathrm{Mt}\right)\right)\right] \\
+\left[\mathrm { c } * \left(\frac{1}{2}[\exp (2 \eta \sqrt{\mathrm{Mt}}) \operatorname{erfc}(\eta+\sqrt{\mathrm{Mt}})+\exp (-2 \eta \sqrt{\mathrm{Mt}}) \operatorname{erfc}(\eta-\sqrt{\mathrm{Mt}})]\right.\right. \\
+\left(\frac{\exp (\mathrm{at})}{2}[\exp (2 \eta \sqrt{\operatorname{Prat}}) \operatorname{erfc}(\eta \sqrt{\operatorname{Pr}}+\sqrt{\mathrm{at}})+\exp (-2 \eta \sqrt{\operatorname{Prat}}) \operatorname{erfc}(\eta \sqrt{\operatorname{Pr}}-\sqrt{\mathrm{at}})]\right) \\
-\left(\frac{\exp (\mathrm{at})}{2}[\exp (2 \eta \sqrt{(\mathrm{M}+\mathrm{a}) \mathrm{t}}) \operatorname{erfc}(\eta+\sqrt{(\mathrm{M}+\mathrm{a}) \mathrm{t}})+\exp (-2 \eta \sqrt{(\mathrm{M}+\mathrm{a}) \mathrm{t}}) \operatorname{erfc}(\eta-\sqrt{(\mathrm{M}+\mathrm{a}) t})]\right) \\
+(\operatorname{erfc}(\eta \sqrt{\operatorname{Pr}})) \\
+\mathrm{a} *\left(\frac{\mathrm{t}}{2}[\exp (2 \eta \sqrt{\mathrm{Mt}}) \operatorname{erfc}(\eta+\sqrt{\mathrm{Mt}})+\exp (-2 \eta \sqrt{\mathrm{Mt}}) \operatorname{erfc}(\eta-\sqrt{\mathrm{Mt}})]\right)
\end{gathered}
$$




$$
\begin{aligned}
& \left.-\frac{\eta \sqrt{t}}{2 \sqrt{M}}[\exp (-2 \eta \sqrt{\mathrm{Mt}}) \operatorname{erfc}(\eta-\sqrt{\mathrm{Mt}})-\exp (2 \eta \sqrt{\mathrm{Mt}}) \operatorname{erfc}(\eta+\sqrt{\mathrm{Mt}})]\right) \\
& \left.\left.-\mathrm{a} *\left(\mathrm{t}\left(\left(1+2 \eta^{2} \operatorname{Pr}\right) \operatorname{erfc}(\eta \sqrt{\operatorname{Pr}})-\frac{2 \eta \sqrt{\operatorname{Pr}}}{\sqrt{\pi}} \exp \left(-\eta^{2} \operatorname{Pr}\right)\right)\right)\right)\right] \\
& +d^{*}\left[\frac{1}{2}[\exp (2 \eta \sqrt{\mathrm{Mt}}) \operatorname{erfc}(\eta+\sqrt{\mathrm{Mt}})+\exp (-2 \eta \sqrt{\mathrm{Mt}}) \operatorname{erfc}(\eta-\sqrt{\mathrm{Mt}})]\right. \\
& +\frac{\exp (\mathrm{bt})}{2}[\exp (2 \eta \sqrt{\mathrm{Sc}(\mathrm{bt})}) \operatorname{erfc}(\eta \sqrt{\mathrm{Sc}}+\sqrt{\mathrm{bt}})+\exp (-2 \eta \sqrt{\mathrm{Sc} \mathrm{bt}}) \operatorname{erfc}(\eta \sqrt{\mathrm{Sc}}-\sqrt{\mathrm{bt}})] \\
& -\frac{\exp (b t)}{2}[\exp (2 \eta \sqrt{(\mathrm{M}+\mathrm{b}) \mathrm{t}}) \operatorname{erfc}(\eta+\sqrt{(\mathrm{M}+\mathrm{b}) \mathrm{t}})+\exp (-2 \eta \sqrt{(\mathrm{M}+\mathrm{b}) \mathrm{t}}) \operatorname{erfc}(\eta-\sqrt{(\mathrm{M}+\mathrm{b}) \mathrm{t}})] \\
& -[\operatorname{erfc}(\eta \sqrt{\mathrm{Sc}})]] \\
& \text { where } \quad \mathrm{a}=\frac{\mathrm{M}}{\operatorname{Pr}-1}, \quad \mathrm{~b}=\frac{\mathrm{M}}{\mathrm{Sc}-1}, \mathrm{c}=\frac{\mathrm{Gr}}{\mathrm{a}^{2}(1-\mathrm{Pr})}, \mathrm{d}=\frac{\mathrm{Gc}}{\mathrm{b}(1-\mathrm{Sc})} \quad \text { and } \quad \eta=\frac{\mathrm{Y}}{2 \sqrt{\mathrm{t}}}
\end{aligned}
$$

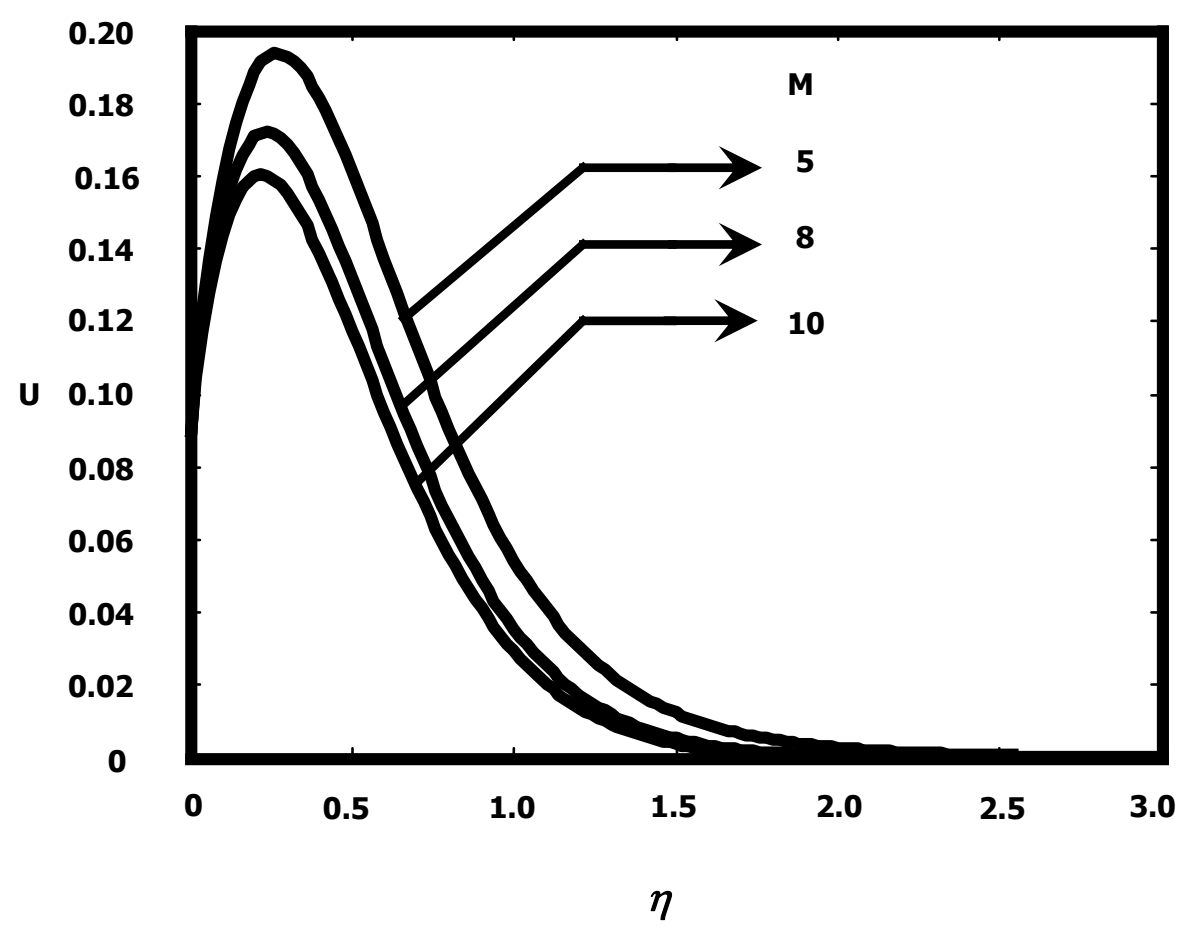

Figure 1. Velocity profiles for different M.

\section{RESULTS AND DISCUSSION}

For physical understanding of the problem, numerical computations are carried out for different physical parameters Gr, Gc, Sc, Pr, M and t upon the nature of the flow and transport. The value of the Schmidt number Sc is 
taken to be 2.01 , which corresponds to water vapour. The numerical values of the velocity are computed for different physical parameters like the Prandtl number, thermal Grashof number, mass Grashof number, Schmidt number and time. Figure 1 illustrates the effects of the magnetic field parameter on the velocity when $(\mathrm{M}=5,8,10), \mathrm{Gr}=\mathrm{Gc}=5, \mathrm{Pr}=7$ and $t=0.3$. It is observed that the velocity increases with decreasing values of the magnetic field parameter. This shows that the increase in the magnetic field parameter leads to a fall in the velocity. This agrees with the expectations, since the magnetic field exerts a retarding force on the free convective flow past the parabolic started vertical plate.

Figure 2 presents the flow structure of the velocity profiles with times $(t=0.3,0.4,0.6,0.8), \mathrm{M}=4, \mathrm{Gr}=\mathrm{Gc}=5, \mathrm{Pr}=7$. Indeed, it maximizes the effects of magnetic flow field that the velocity increases with increasing values of $t$ from the trend lines. Figure 3 demonstrates the inlet flow effects of different thermal Grashof numbers $(\mathrm{Gr}=2,5)$, mass Grashof numbers ( $\mathrm{Gc}=5,10)$ with the corresponding Prandtl number $=7$ and $M=4$ on the velocity at time $t=0.3$. It can be observed that the velocity increases with increasing values of the thermal Grashof number as well as mass Grashof number.

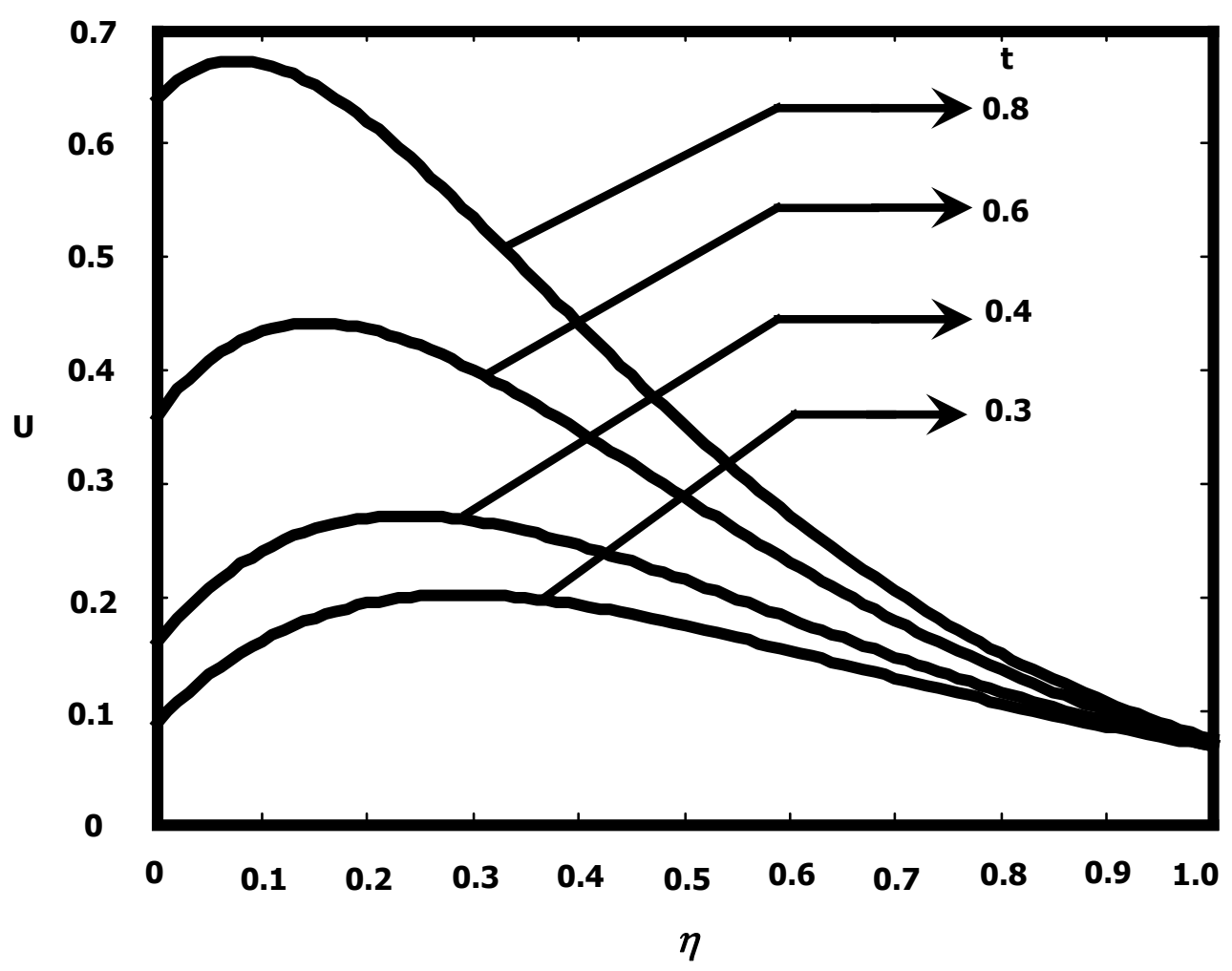

Figure 2. Velocity profiles for different $\mathrm{t}$. 


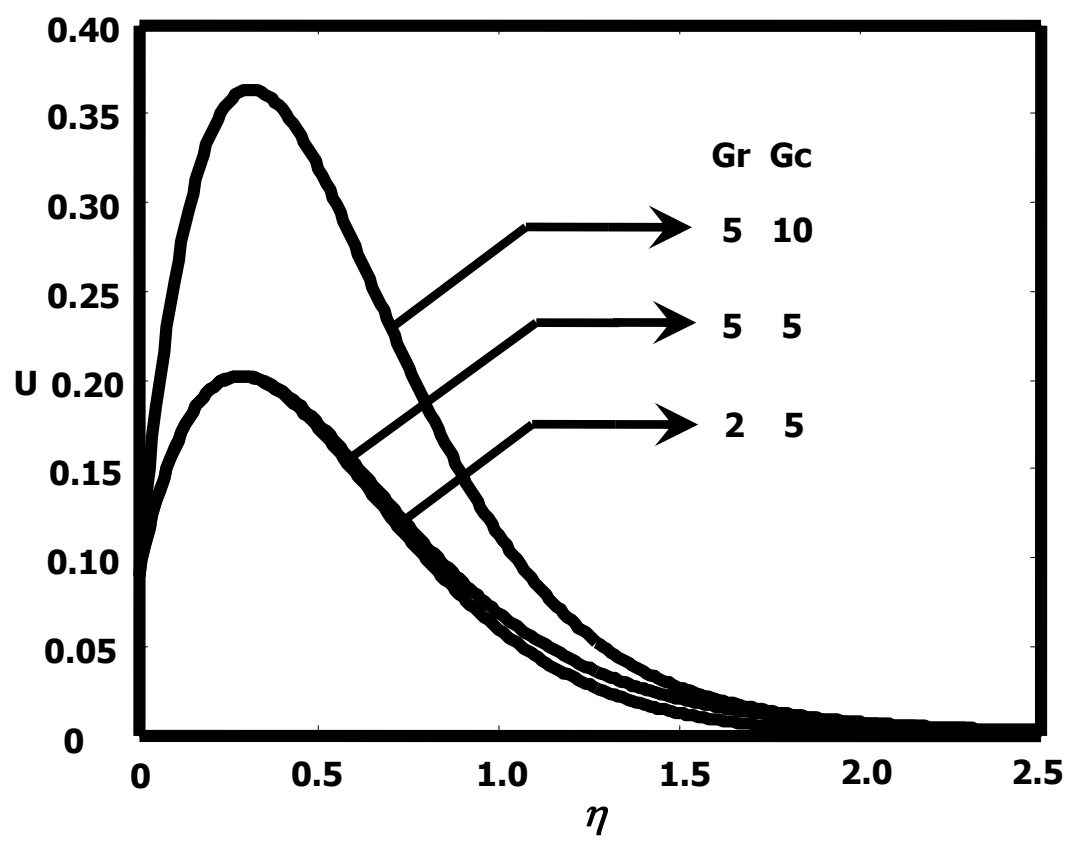

Figure 3. Velocity profiles for different Gr \& Gc.

\section{CONCLUSIONS}

The theoretical analysis of magnetohydrodynamic flow past a parabolic motion of an infinite vertical plate with variable temperature and uniform mass diffusion has been analysed. The dimensionless governing equations are solved by the usual Laplace transform technique. The effect of velocity for different parameters like the thermal Grashof number, mass Grashof number and magnetic field parameters and time parameter are studied graphically. We conclude that the velocity increases with increasing values of Gr, Gc and t. But the trend line is reversed with respect to the magnetic field parameter.

\section{ACKNOWLEDGEMENTS}

The author would like to thank Manonmaniam Sundaranar University India for providing financial support and laboratory facilities.

\section{REFERENCES}

[1] Chenna Kesavaiah D, Sudhakaraiah A. Effects of heat and mass flux to MHD flow in vertical surface with radiation absorption. Scholars Journal of Engineering and Technology. 2014;2:219-25.

[2] Satya Narayana PV, Ramireddy G, Venkataramana S. Hall current effects on free-convection MHD flow past a porous plate. International Journal of Automotive and Mechanical Engineering. 2011;3:350-63. 
[3] Muthucumaraswamy R, Dhanasekar N, Easwara Prasad G. Mass transfer effects on accelerated vertical plate in a rotating fluid with first order chemical reaction. Journal of Mechanical Engineering and Sciences. 2012;3:346-55.

[4] Muthucumaraswamy R, Radhakrishnan M. Chemical reaction effects on flow past an accelerated vertical plate with variable temperature and mass diffusion in the presence of magnetic field. Journal of Mechanical Engineering and Sciences. 2012;3:251-60.

[5] Singh A, Singh J. Mass transfer effects on the flow past an accelerated vertical plate with constant heat flux. Astrophysics and Space Science. 1983;97:57-61.

[6] Attia H, Kotb N. MHD flow between two parallel plates with heat transfer. Acta mechanica. 1996;117:215-20.

[7] Manay E, Ozceyhan V, Sahin B, Gunes S. Edge length effect of bluff bodies on flow structure. International Journal of Automotive and Mechanical Engineering. 2014;9:1793-801.

[8] Gupta A, Popp I, Soundalgekar V. Free convection effects on the flow past an accelerated vertical plate in an incompressible dissipative fluid. Revue Roumaine des Sciences Techniques Serie de Mecanique Appliquee. 1979;24:561-8.

[9] Raptis A, Tzivanidis G, Perdikis C. Hydromagnetic free convection flow past an accelerated vertical infinite plate with variable suction and heat flux. Letters in Heat and Mass Transfer. 1981;8:137-43.

[10] Soundalgekar V. Effects of mass transfer on flow past a uniformly accelerated vertical plate. Letters in Heat and Mass Transfer. 1982;9:65-72.

[11] Jha BK, Prasad R. Free-convection and mass transfer effects on the flow past an accelerated vertical plate with heat sources. Mechanics Research Communications. 1990;17:143-8.

[12] Agrawal A, Samria N, Gupta S. Study of heat and mass transfer past a parabolic started infinite vertical plate. Journal of Energy, Heat and Mass Transfer. 1999;21:67-76.

[13] Manning R, Ewing, J. (2009). RACQ Vehicles Technologies. Temperatures in cars survey. RACQ Vehicles Technologies. 2009:1-21.

[14] Papuga J. A survey on evaluating the fatigue limit under multiaxial loading. International Journal of Fatigue. 2011;33:153-65.

[15] Wang J, Lu MX, Zhang L, Chang W, Xu LN, Hu LH. Effect of welding process on the microstructure and properties of dissimilar weld joints between low alloy and duplex stainless steel. International Journal of Minerals, Metallurgy and Materials. 2012;19:518-24.

\section{NOMENCLATURES}

A Constants

$B_{0} \quad$ external magnetic field

$C$ dimensionless concentration

$C_{p} \quad$ specific heat at constant pressure $J \mathrm{~kg}^{-1} \mathrm{k}$

$C^{\prime} \quad$ species concentration in the fluid $\mathrm{k} \mathrm{g} \mathrm{m}^{-3}$

$C_{w}^{\prime} \quad$ wall concentration in the fluid

$C_{\infty}^{\prime}$ concentration in the fluid far away from the plate 
$D$ mass diffusion coefficient $m^{2} s^{-1}$

Gc mass Grashof number

Gr thermal Grashof number

$g \quad$ acceleration due to gravity $m^{2} s^{-1}$

$k \quad$ thermal conductivity $W m^{-1} k^{-1}$

$\mathrm{Pr}$ Prandtl number

Sc Schmidt number

$T$ temperature of the fluid near the plate

$T_{w} \quad$ temperature of the plate

$T_{\infty} \quad$ temperature of the fluid far away from the plate

$t$ dimensionless time

$t^{\prime}$ time $\mathrm{s}$

$u \quad$ velocity of the fluid in the $x^{\prime}$ - direction $\mathrm{ms}^{-1}$

$u_{0} \quad$ velocity of the plate $m s^{-1}$

$U$ dimensionless velocity

y co-ordinate axis normal to the plate $\mathrm{m}$

$Y$ dimensionless coordinate axis normal to the plate

M magnetic field parameter

\section{Greek Symbols}

$\beta \quad$ volumetric coefficient of thermal expansion $K^{-1}$

$\beta^{*} \quad$ volumetric coefficient of expansion with concentration $K^{-1}$

$\mu \quad$ coefficient of viscosity $R a s$

$v \quad$ kinematic viscosity $m^{2} s^{-1}$

$\sigma \quad$ electrical conductivity

$\rho$ density of the fluid $\mathrm{kg} \mathrm{m}^{-3}$

$\tau \quad$ dimensionless skin-friction $\mathrm{kg} \mathrm{m}^{-3}$

$\theta$ dimensionless temperature

$\eta \quad$ similarity parameter

erfc complementary error function

\section{Subscripts}
$w$ conditions at the wall
$\infty$ free stream conditions 